\title{
Uncertain portfolio optimization problem with liquidity and diversification
}

\author{
Ranran Zhang ${ }^{1}$ and $\mathrm{Bo} \mathrm{Li}^{2}$ \\ ${ }^{1}$ Nanjing University of Finance and Economics - Xianlin Campus \\ ${ }^{2}$ Nanjing University of Finance and Economics
}

November 23, 2020

\begin{abstract}
This paper deals with a portfolio selection problem with uncertain returns. Here, the returns of the assets are regarded as uncertain variables which are estimated by experienced experts. First, an uncertain mean-variance-entropy model for portfolio selection problem is presented by taking into account four criteria viz., return, risk, liquidity and diversification degree of portfolio. In the proposed model, the investment return is quantified by uncertain expected value, the investment risk is characterized by uncertain variance and entropy is used to measure the diversification degree of portfolio. Moreover, different from the previous bi-objective optimization model, our model achieves both the maximum return and the minimum risk in a single objective form by introducing a risk aversion factor and the dimensional influence caused by different units is eliminated by normalization. Then, two auxiliary portfolio selection models are transformed into different equivalent deterministic models. Finally, a numerical simulation is given to verify the practicability of our model.
\end{abstract}

\section{Hosted file}

uncertain portfolio optimization problem with liquidty and diversification.pdf available at https://authorea.com/users/378164/articles/494709-uncertain-portfolio-optimizationproblem-with-liquidity-and-diversification

\section{Hosted file}

zrr2020.8.13.tex available at https://authorea.com/users/378164/articles/494709-uncertainportfolio-optimization-problem-with-liquidity-and-diversification 
figures/11/11-eps-converted-to.pdf 
figures/22/22-eps-converted-to.pdf 